\title{
Pain relief after shoulder surgery - what happens when the block wears off
}

\section{Mclvor, J Mitchell, J Ramsey \\ Department of Anaesthesia \& Intensive Care Medicine, University Hospital Ayr, Ayr, KA6 6DX, Scotland}

\section{Introduction}

- Brachial plexus blockade is widely recognised as an effective form of analgesia for shoulder surgery. It has enabled surgeons to perform complex procedures on day case patients.

- When a single injection nerve block wears off pain management becomes a major challenge ${ }^{1}$. Our previous audit found that a third of patients reported severe pain with movement after nerve block regression.

- We introduced the option of prescribing short-acting oxycodone to supplement discharge analgesia; and a patient information leaflet to give advice on pain relief. Further evaluation was carried out.

\section{Method}

- Patients undergoing elective day case shoulder surgery who had a brachial plexus block performed were included.

- The surgical procedure, type of block, time of insertion, drug dose, drug volume and additives were recorded.

- Patients received a follow-up telephone call 2-4 days postoperatively.

- Patients were asked when they first noted return of normal sensation. Pain scores were obtained during rest and movement using numerical rating score (NRS) and verbal rating score (VRS) Additional information was sought regarding residual nerve symptoms.

- Post-operative analgesia requirements were recorded and patients reported on the adequacy of pain killers supplied. Patients were also asked if they would have a nerve block again.

\section{Results}

- 30 patients were identified over a 4 month period. 22 patients were contacted by telephone. 8 were lost to follow-up.

- All patients had an ultrasound guided interscalene block performed with $15-20 \mathrm{ml}$ of $0.5 \%$ Levobupivicaine. The average duration of block was $25.8 \pm 6.2$ hours. Perineural clonidine was included in $18 / 22$ blocks. The addition of perineural clonidine extended the block time by approximately 2 hours. There were no failed blocks recorded.

- 6/22 patients reported severe pain at rest. 10/22 experienced severe pain during movement. NRS and VRS pain scores correlated.

- $8 / 22$ felt they needed stronger pain relief. Only 3 patients had been prescribed oxycodone for discharge. Despite this, all oxycodone patients reported severe pain during rest and movement.

- All but 1 patient stated that they would have a repeat nerve block.

- All patients were either satisfied or very satisfied with their day surgery experience.

\footnotetext{
References

1. Beecroft C, Coventry D. Anaesthesia for shoulder surgery, Continuing Education in Anaesthesia, Critical Care \& Pain 2008 Volume 8 Number 6

2. Giraudon I, Lowitz K, Dargan PI et al. Prescription opioid abuse in the UK. Br J Clin Pharmacol 2013;76:823-824

3. Fredrickson $\mathrm{M}$ et al Postoperative analgesia for shoulder surgery: a critical appraisal and review of current techniques. Anaesthesia 2010;65;608-624
}
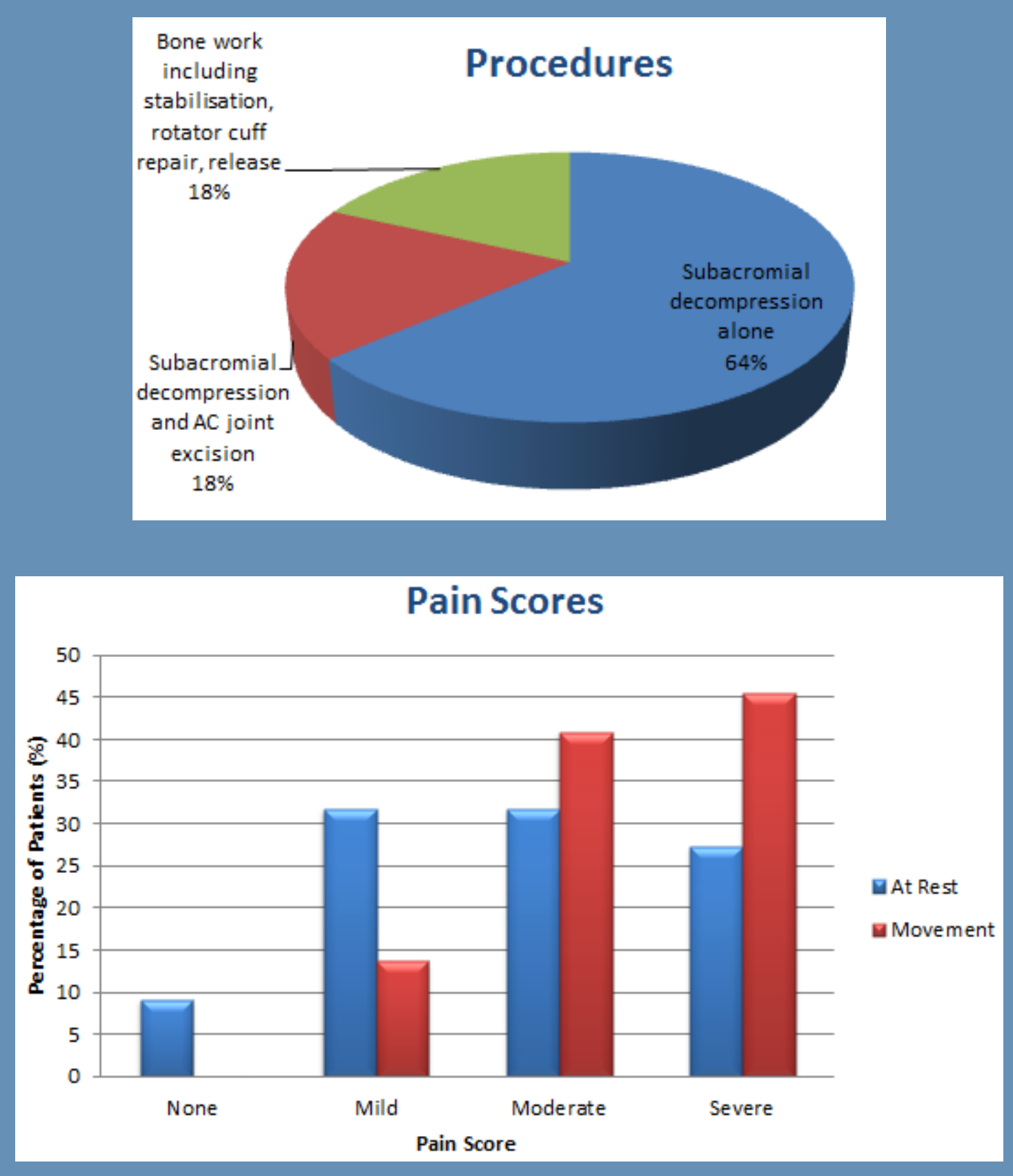

\section{Discussion}

- We have demonstrated that our block service is effective with no failed blocks. However, once pain relief provided by the nerve block wore off almost half of patients reported severe pain, and a third reported inadequate analgesia.

- Despite the introduction of oxycodone to supplement analgesia, and a patient information leaflet to give advice on pain relief, patients often did not take analgesia as prescribed.

- Routine prescription of oxycodone for discharge after shoulder surgery is an option, however only a third of patients may actually require this. This may become problematic, particularly given the current challenges with opioid prescribing patterns ${ }^{2}$.

\section{Conclusion}

- Single injection brachial plexus blocks are very effective but have limited duration. The use of continuous interscalene nerve catheters may improve analgesia ${ }^{3}$. However, this has technical and logistical challenges, with limited additional duration due to elastomeric pump capacity.

- A key consideration is the use of preventive analgesia, which involves effective pain relief in the perioperative and post discharge period. Strong opioid analgesia should be routinely prescribed for complex shoulder procedures such as rotator cuff repair or shoulder stabilisation, as part of a multimodal regime.

- Routine telephone follow up is an important adjunct to assess adequacy of postoperative analgesia and recovery. It will provide advice and support to improve pain management, record and troubleshoot any complications associated with nerve blockade. 